TRANSACTIONS OF THE

AMERICAN MATHEMATICAL SOCIETY

Volume 352, Number 5, Pages 2077-2086

S 0002-9947(99)02587-8

Article electronically published on November 17, 1999

\title{
A NOTE ON ELLIPTIC K3 SURFACES
}

\author{
JONGHAE KEUM
}

\begin{abstract}
We study the relationship between an elliptic fibration on an elliptic K3 surface and its Jacobian surface. We give an explicit description of the Picard lattice of the Jacobian surface. Then we use the description to prove that certain K3 surfaces do not admit a non-Jacobian fibration. Moreover, we obtain an inequality involving the determinant of the Picard lattice and the number of components of reducible fibres, which implies, among others, that if an elliptic K3 surface has Picard lattice with relatively small determinant, then every elliptic fibration on it must have a reducible fibre. Some examples of K3 surfaces are discussed.
\end{abstract}

\section{INTRODUCTION}

By a K3 surface we mean a simply connected projective complex surface with trivial canonical bundle. A K3 surface is called elliptic if it admits an elliptic fibration. For example, any K3 surface with Picard number $\geq 5$ is always elliptic. In this paper we study some properties of elliptic fibrations on K3 surfaces and their Jacobian surfaces. To be more precise, let $f: S \rightarrow \mathbb{P}^{1}$ be an elliptic fibration on a K3 surface $S$. Let $l$ be its multisection index, i.e. the positive minimum possible intersection number of a fibre and a divisor. The fibration has a section if and only if $l=1$. We call the number $k=\Sigma\left(m_{v}-1\right)$ the index of reducibility of $f$, where $m_{v}$ is the number of components of a singular fibre $F_{v}$. Denote by $J(S)$ the Jacobian surface corresponding to $f$, which is known to be an elliptic K3 surface with the same Picard number and singular fibres of the same type as the surface $f: S \rightarrow \mathbb{P}^{1}$ (cf. [3]).

First we give an explicit way of computing the Picard lattice Pic $(J(S))$ of $J(S)$ in terms of $\operatorname{Pic}(S)$ and a fibre of $f$, proving that $\operatorname{Pic}(J(S))$ is an over-lattice of $\operatorname{Pic}(S)$ of index $l$ corresponding to the isotropic element (a fibre) $/ l$ of order $l$ in the discriminant group of $\operatorname{Pic}(S)$ (Lemma 2.1). This result, essentially follows from the work of Mukai [8] on moduli spaces of stable sheaves on K3 surfaces, turns out to be useful. It can be used to compute the possible values of $l$ (Corollary 2.2) and to prove that certain K3 surfaces do not admit a non-Jacobian fibration (Theorem 2.3). Section 2 ends with an example of a Kummer surface with an elliptic fibration whose Jacobian surface fails to be Kummer (Proposition 2.5).

Using Lemma 2.1, the theory of Mordell-Weil lattices due to Shioda [12, 13 and Rogers' bound for sphere packing (cf. 2]) we obtain an inequality involving $\operatorname{det} \operatorname{Pic}(S)$, multisection index $l$ and index of reducibility $k$ (Theorems 3.1, 3.3).

Received by the editors October 22, 1997.

2000 Mathematics Subject Classification. Primary 14J28, 14J27, 11 H31.

Key words and phrases. Elliptic K3 surface, Jacobian surface, Picard lattice, lattice packing.

The research was supported by the Korea Research Foundation (1998) and GARC. 
It follows e.g. that if $\operatorname{det} \operatorname{Pic}(S)$ is small enough, then every elliptic fibration on $S$ must have a reducible fibre (Corollary 3.4). Some examples of K3 surfaces are discussed at the end of Section 3.

Finally the author would like to thank Professor Shioda for valuable conversations, which initiated this paper, and also Professor Dolgachev for helpful discussions, especially for the proof of Lemma 2.1.

\section{Mordell-Weil lattices}

Recently T. Shioda has done a systematic study of Mordell-Weil lattices in the case of elliptic surfaces. In this section, we review the definition and some basic results. The main reference is [12] or [13].

We assume that the ground field is $\mathbb{C}$, although everything in this section is true in an arbitrary characteristic.

Let $K=\mathbb{C}(C)$ be the function field of a smooth complex projective curve $C$.

Let $E$ be an elliptic curve defined over $K$ with a $K$-rational point, and let $E(K)$ denote the group of $K$-rational points of $E$, with origin $O$. The Kodaira-Néron model of $E / K$ is an elliptic surface $f: S \rightarrow C$ with a section, where $S$ is a smooth complex projective surface and $f$ is a relatively minimal fibration with the generic fibre $E$. So, the group $E(K)$ of $K$-rational points of $E$ can be identified with the group of sections of $f$. For $P \in E(K),(P)$ will denote the image curve of $P: C \rightarrow S$.

Assume throughout that $f$ has at least one singular fibre. Then $E(K)$ is finitely generated (Mordell-Weil theorem) and the Néron-Severi group $N S(S)$ of $S$, the group of divisors modulo algebraic equivalence, becomes an integral lattice of finite rank with respect to the intersection pairing $D . D^{\prime}$. Let $T$ denote the sublattice of $N=N S(S)$ generated by zero section $(O)$, a fibre $F$ and the irreducible components of fibres. Then $T$ is a direct sum:

$$
T=(\mathbb{Z}(O)+\mathbb{Z} F) \oplus\left(\bigoplus_{v \in R} T_{v}\right)
$$

where $R=\left\{v \in C: F_{v}=f^{-1}(v)\right.$ is reducible $\}$ and $T_{v}$ is generated by the irreducible components of $F_{v}$ not meeting the zero section $(O)$.

The map $P \rightarrow(P) \bmod T$ induces a group isomorphism :

$$
E(K) \cong N S(S) / T
$$

and then a unique homomorphism :

$$
\phi: E(K) \rightarrow N S(S) \otimes \mathbb{Q}
$$

such that

$$
\phi(P)=(P) \bmod T \otimes \mathbb{Q}, \quad i m(\phi) \perp T .
$$

For $P, P^{\prime} \in E(K)$, let

$$
\left\langle P, P^{\prime}\right\rangle=-\phi(P) . \phi\left(P^{\prime}\right) .
$$

Then with this pairing the group $E(K) / E(K)_{\text {tor }}$ becomes a positive definite lattice, called the Mordell-Weil lattice of $E / K$ or of $f: S \rightarrow C$. Mordell-Weil lattices are not integral lattices in general. 
The above height pairing can be written as follows:

$$
\left\langle P, P^{\prime}\right\rangle=\chi+P . O+P^{\prime} . O-P . P^{\prime}-\sum_{v \in R} \operatorname{contr}_{v}\left(P, P^{\prime}\right) .
$$

Here $\chi$ is the algebraic Euler characteristic of the surface $S, P . Q=(P) \cdot(Q)$, and the local contribution term $\operatorname{contr}_{v}\left(P, P^{\prime}\right)$ is a nonnegative rational number, which is nonzero only if both $P$ and $P^{\prime}$ pass through non-identity components of $F_{v}$; it is indeed the $(i, j)$-entry of the matrix $\left(-A_{v}^{-1}\right)$, where $A_{v}$ is the intersection matrix of non-identity components of $F_{v}$, and $P$ and $P^{\prime}$ meet the $i$-th and $j$-th components, respectively.

The subgroup $E(K)^{0}$ of $E(K)$ consisting of those sections meeting the identity component of every fibre is a torsion-free subgroup of $E(K)$ of finite index, and becomes a positive definite even lattice, called the narrow Mordell-Weil lattice of $E / K$ or of $f: S \rightarrow C$. This lattice is isomorphic via the map $\phi$ to the opposite lattice $T^{\perp}(-1)$ of the orthogonal complement $T^{\perp}$ of $T$ in the Neron-Severi lattice $N S(S)$.

Note that

$$
\begin{gathered}
\left\langle P, P^{\prime}\right\rangle=\chi+P . O+P^{\prime} . O-P . P^{\prime} \in \mathbb{Z} \quad \text { if } P \text { or } P^{\prime} \in E(K)^{0}, \\
\langle P, P\rangle=2 \chi+2 P . O \geq 2 \chi \quad \text { for } \quad P \in E(K)^{0}, P \neq O .
\end{gathered}
$$

The invariants of Mordell-Weil lattices can be expressed in terms of geometric data of the surface $S$. Let $M=E(K)^{0}$ be the narrow Mordell-Weil lattice of $f: S \rightarrow C$. Then

$$
\begin{gathered}
\operatorname{rank} M=\rho(S)-2-\sum_{v \in R}\left(m_{v}-1\right), \\
\operatorname{det} M=\nu^{2}|\operatorname{det} N S(S) / \operatorname{det} T|, \quad \nu=\left[E(K): E(K)^{0}\right], \\
\mu(M)=2 \chi+2 \min \left\{P . O: P \in E(K)^{0}, P \neq O\right\} \geq 2 \chi,
\end{gathered}
$$

where $\rho(S)=\operatorname{rank} N S(S)$ is the Picard number of $S$ and $m_{v}$ is the number of irreducible components of the singular fibre $F_{v}$, and $\mu(M)$ is the square of the minimal norm of $M$.

The following lemma will be used in Section 3.

Lemma 1.1. With the same notation, we have

$$
\nu^{2} /|\operatorname{det} T| \leq|\operatorname{det} T| \leq 2^{k},
$$

where $k=\sum_{v \in R}\left(m_{v}-1\right)$.

Proof. Note that there is an embedding :

$$
E(K) / E(K)^{0} \rightarrow \Pi_{v}\left(F_{v}^{*} / F_{v}^{* 0}\right)
$$

where $F_{v}^{*}$ and $F_{v}^{* 0}$ are the smooth part and the identity component of $F_{v}$, resp., and see that

$$
\nu \leq \Pi_{v}\left|F_{v}^{*} / F_{v}^{* 0}\right|=\Pi_{v}\left(\operatorname{det} T_{v}\right)=\operatorname{det} T .
$$

Next it is easy to see that $\operatorname{det} T$ takes its maximum $2^{k}$ only when $m_{v}=2$ for all $v \in R$. 


\section{Elliptic K3 SURfaces AND their JACOBian SURFACES}

Let $f: S \rightarrow \mathbb{P}^{1}$ be an elliptic K3 surface. It is known (cf. [3]) that the Jacobian surface $J(S)$ of $S$ is an elliptic K3 surface and that $J(S)$ and $S$ have the same Picard number and singular fibres of the same type.

Let $F$ be a general fibre of $f$. Then the set

$$
\{D . F: D \in \operatorname{Pic}(S)\}
$$

is an ideal of $\mathbb{Z}$. The positive generator $l$ of this ideal is called the multisection index of $f: S \rightarrow \mathbb{P}^{1}$. Note that the element $F / l$ belongs to the dual lattice $\operatorname{Pic}(S)^{*}$ and gives an isotropic element of order $l$ in the discriminant group of $\operatorname{Pic}(S)$.

The following result, which contains a relationship between the Picard lattices of the surface $S$ and its Jacobian surface $J(S)$, will serve as a useful tool for the rest of the paper.

Lemma 2.1. Let $f: S \rightarrow \mathbb{P}^{1}$ be an elliptic $K 3$ surface with multisection index l. Let $F$ be the divisor class of a fibre. Then Pic $(S)$ embeds in $\operatorname{Pic}(J(S))$ as a sublattice of index $l$ and the quotient Pic $(J(S)) / \operatorname{Pic}(S)$ is a cyclic group of order $l$ generated by $F / l$. (That is, Pic $(J(S))$ is generated as a lattice by $\operatorname{Pic}(S)$ and $F / l$.) In particular,

$$
\operatorname{det} \operatorname{Pic}(S)=l^{2} \operatorname{det} \operatorname{PicJ}(S) .
$$

Proof. This lemma essentially follows from Mukai 8 .

Let $\tilde{H}(S, \mathbb{Z})$ be the cohomology ring

$$
H^{*}(S, \mathbb{Z})=H^{0}(S, \mathbb{Z}) \oplus H^{2}(S, \mathbb{Z}) \oplus H^{4}(S, \mathbb{Z})
$$

with the following bilinear form and Hodge structure of weight 2 (see 8 ]):

(i) The bilinear form ( . ) on $H^{*}(S, \mathbb{Z})$ is defined by

$$
\left((r, D, s) \cdot\left(r^{\prime}, D^{\prime}, s^{\prime}\right)\right)=-r s^{\prime}+D \cdot D^{\prime}-r^{\prime} s \in \mathbb{Z}
$$

for every $(r, D, s)$ and $\left(r^{\prime}, D^{\prime}, s^{\prime}\right)$ in $H^{*}(S, \mathbb{Z})$, where we identify $H^{0}(S, \mathbb{Z})$ and $H^{4}(S, \mathbb{Z})$ with $\mathbb{Z}$ by the fundamental cycle and cocycle.

(ii) The Hodge decomposition of $\tilde{H}(S, \mathbb{C})=\tilde{H}(S, \mathbb{Z}) \otimes \mathbb{C}$ is defined by

$$
\begin{gathered}
\tilde{H}^{2,0}(S, \mathbb{C})=H^{2,0}(S, \mathbb{C}), \\
\tilde{H}^{0,2}(S, \mathbb{C})=H^{0,2}(S, \mathbb{C}), \\
\tilde{H}^{1,1}(S, \mathbb{C})=H^{0}(S, \mathbb{C}) \oplus H^{1,1}(S, \mathbb{C}) \oplus H^{4}(S, \mathbb{C}) .
\end{gathered}
$$

Let $v=(0, F,-l)$ be an element of $\tilde{H}(S, \mathbb{Z})$ and let $M(v)$ be the moduli space of (stable coherent) sheaves $\mathcal{E}$ on $S$ with respect to a fixed embedding $S \rightarrow \mathbb{P}^{N}$ with $\operatorname{rank} \mathcal{E}=0, c_{1}(\mathcal{E})=F$ and $c_{2}(\mathcal{E})=l$, i.e.

$$
\left(\operatorname{rank} \mathcal{E}, c_{1}(\mathcal{E}), \operatorname{rank} \mathcal{E}+1 / 2 c_{1}(\mathcal{E})^{2}-c_{2}(\mathcal{E})\right)=v
$$

Since $v$ is primitive (i.e. not divisible in $\tilde{H}(S, \mathbb{Z})$ ) and isotropic (i.e. $\left.v^{2}=0\right), M(v)$ is smooth and 2-dimensional.

We identify the Jacobian surface $J(S)$ of $f: S \rightarrow \mathbb{P}^{1}$ with the compactification of the Picard variety of the generic fibre of $f$ or the relative Picard variety

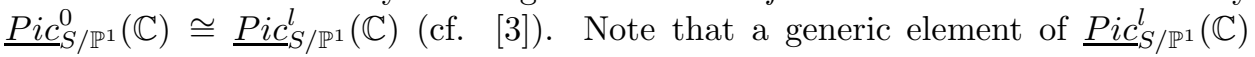
can be represented by a pair $(Z, F)$, where $Z$ is a divisor of degree $l$ on the fibre $F$. Let $i: F \rightarrow S$ be the inclusion morphism. It is easy to check that for each 
$(Z, F) \in \underline{P i c}_{S / \mathbb{P}^{1}}^{l}(\mathbb{C})$ the sheaf $i_{*} \mathcal{O}_{F}(Z)$ on $S$ belongs to the moduli space $M(v)$.

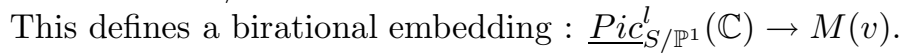

Now by a result of Mukai (Theorem 1.4 and Remark, [8]) the K3 surface $J(S)$ is the compactification of a component of $M(v)$ and there is an isomorphism of Hodge structures between $H^{2}(J(S), \mathbb{Z})$ and $v^{\perp} / \mathbb{Z} v$ which is compatible with the intersection pairing on $H^{2}(J(S), \mathbb{Z})$ and the bilinear form on $v^{\perp} / \mathbb{Z} v$ induced by that on $\tilde{H}(S, \mathbb{Z})$. From this, we see that

$$
\begin{gathered}
\operatorname{Pic}(J(S)) \cong\left(v^{\perp} \cap \tilde{H}^{1,1}(S, \mathbb{C})\right) / \mathbb{Z} v \\
=\{(-D \cdot F / l, D, b) \in \tilde{H}(S, \mathbb{Z}): D \in \operatorname{Pic}(S), b \in \mathbb{Z}\} / \mathbb{Z} v \\
=\{\{(-D \cdot F / l, D, 0): D \in \operatorname{Pic}(S)\}+\mathbb{Z}(0,0,1)\} / \mathbb{Z} v .
\end{gathered}
$$

Now it is easy to see that the map

$$
\psi: \operatorname{Pic}(S) \rightarrow \operatorname{Pic}(J(S)), \quad \psi(D)=(-D . F / l, D, 0)
$$

is an isometric embedding. Finally we see that

$$
l(0,0,1) \equiv \psi(F) \quad \bmod v .
$$

Thus

$$
\operatorname{Pic}(J(S)) \cong \operatorname{Pic}(S)+\mathbb{Z} F / l \subset \operatorname{Pic}(S)^{*} .
$$

The result follows from the primitivity of $F$ in $\operatorname{Pic}(S)$.

Remark. (i) In [1] Belcastro has proved a similar result in a slightly different way; she applied the result of Mukai to the moduli space of stable vector bundles $\mathcal{E}$ on the surface $S$ with respect to a fixed embedding $S \rightarrow \mathbb{P}^{N}$ with $\operatorname{rank} \mathcal{E}=l, c_{1}(\mathcal{E})=F$ and $c_{2}(\mathcal{E})=l$ (the Mukai vector in this case is $v=(l, F, 0)$ ). We believe our result is a bit more precise.

(ii) We point out that Lemma 2.1 can not be generalized directly to general elliptic surfaces. In fact, every Enriques surface is elliptic and its corresponding Jacobian surface is isomorphic to a projective plane with nine points blown up. The Picard lattice of an Enriques surface modulo torsion is even unimodular and of signature $(1,9)$, while the Picard lattice of its Jacobian surface is odd unimodular.

Corollary 2.2. Let $S$ be an algebraic K3 surface.

1. If $\operatorname{det} \operatorname{Pic}(S)$ is square free, then every elliptic fibration on $S$ is a Jacobian fibration, i.e. has a section.

2. If the discriminant group of Pic $(S)$ does not contain an isotropic element of order $l$, then $S$ does not admit an elliptic fibration with multisection index $l$.

Example. $S=$ the Fermat quartic surface in $\mathbb{P}^{3}$.

The transcendental lattice $T(S)=\langle 8\rangle \oplus\langle 8\rangle$ and $\operatorname{det} \operatorname{Pic}(S)=64$. Since there are no isotropic elements of order 4 or 8 in the discriminant group of $\operatorname{Pic}(S)$, the multisection index $l$ of any elliptic fibration must be 1 or 2 .

Now let us consider the following K3 surfaces :

$X_{m}(m=3,4)$, the minimal resolution of the quotient surface

$$
\begin{gathered}
E_{\tau_{m}} \times E_{\tau_{m}} /\left\langle\sigma:\left(z_{1}, z_{2}\right) \rightarrow\left(\tau_{m} z_{1}, \tau_{m}^{-1} z_{2}\right)\right\rangle \\
\left(\tau_{m}=m \text {-th root of } 1\right),
\end{gathered}
$$


where $E_{\tau}$ is the elliptic curve with period $\tau$. These surfaces are singular K3 surfaces whose automorphism groups were computed by Vinberg [14].

As an application of Lemma 2.1, we have the following :

Theorem 2.3. Let $X$ be one of the following three singular K3 surfaces:

$$
X_{3}, \quad X_{4}, \quad K m\left(E_{\tau_{3}} \times E_{\tau_{3}}\right) .
$$

Then every elliptic fibration on $X$ is a Jacobian fibration.

Proof. (i) If $X=X_{3}$, then $\operatorname{det} \operatorname{Pic}(X)=3$, which is square free.

(ii) If $X=X_{4}$, then $\operatorname{det} \operatorname{Pic}(X)=4$ and, since there is no even unimodular lattice of signature $(1,19)$, we are done by Lemma 2.1 .

(iii) If $X=K m\left(E_{\tau_{3}} \times E_{\tau_{3}}\right)$, then $\operatorname{det} \operatorname{Pic}(X)=12$, so we need to rule out the possibility $l=2$.

Suppose $l=2$ for some elliptic fibration on $X$. Then PicJ $(X)$, being an overlattice of $\operatorname{Pic}(X)$ of index 2, corresponds to an isotropic element of order 2 in the discriminant group of $\operatorname{Pic}(X)$. Since the transcendental lattice of $X$ is generated by two elements $e, f$ with $e^{2}=f^{2}=4$, ef $=2$, one can check that there is no such element in the discriminant group of $\operatorname{Pic}(X)$.

Remark. Recently, Nishiyama [10] has classified all possible Jacobian fibrations on each of the three K3 surfaces in Theorem 2.3 (plus two more Kummer surfaces). The above theorem strengthens his result.

On the other hand, there exists an example of a Kummer surface with an elliptic fibration whose Jacobian surface fails to be a Kummer surface.

First, we need the following, which easily follows from Morrison 7, Corollary 4.4].

Lemma 2.4. Let $S$ be an algebraic Kummer surface.

1. If $\rho(S)=20$, then $|\operatorname{det} \operatorname{Pic}(S)| \geq 12$.

2. If $\rho(S)=19$, then $|\operatorname{det} \operatorname{Pic}(S)| \geq 16$.

3. If $\rho(S)=18$, then $|\operatorname{det} \operatorname{Pic}(S)| \geq 16$.

4. If $\rho(S)=17$, then $|\operatorname{det} \operatorname{Pic}(S)| \geq 64$.

Proposition 2.5. Let $S$ be the Kummer surface $K m\left(E \times E^{\prime}\right)$ of the product of non-isogenous elliptic curves $E$ and $E^{\prime}$. Then there exists an elliptic fibration on $S$ whose Jacobian surface is not a Kummer surface.

Proof. Note that the transcendental lattice $T(S)$ and the Picard lattice Pic $(S)$ of $S$ can be computed to be

$$
T(S)=U(2) \oplus U(2), \quad \operatorname{Pic}(S)=D_{8} \oplus D_{8} \oplus U .
$$

Thus

$$
\rho(S)=18, \quad \operatorname{det} \operatorname{Pic}(S)=16 .
$$

We shall find explicitly such an elliptic fibration. Recall that the surface $S$ contains 24 smooth rational curves, 4 from the elliptic curves $E \times$ (a 2-torsion of $E^{\prime}$ ), 4 from (a 2-torsion of $E$ ) $\times E^{\prime}$, and 16 from the resolution of 16 nodes. The configuration of these 24 curves is given in Figure 1.

Denote by $H_{i}(i=1,2,3,4)$ the 4 horizontal curves, by $V_{j}(j=1,2,3,4)$ the 4 vertical ones, and by $N_{i j}$ the curve meeting $H_{i}$ and $V_{j}$. The divisor

$$
2 V_{1}+4 N_{11}+6 H_{1}+5 N_{14}+4 V_{4}+3 N_{44}+2 H_{4}+N_{42}+3 N_{12}
$$




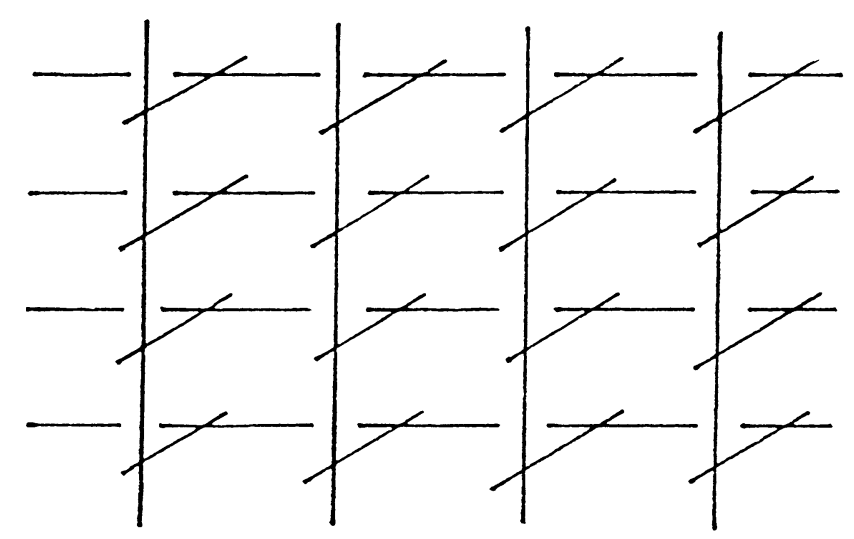

FiguRE 1.

is of type $\tilde{E}_{8}$ and defines an elliptic fibration on $S$. Away from this fibre there are seven curves,

$$
N_{22}, H_{2}, N_{23}, V_{3}, N_{33}, H_{3}, N_{32} \text {. }
$$

These seven curves form a string and hence a part of a fibre of type $\tilde{D}_{8}, \tilde{A}_{7}, \tilde{A}_{8}, \tilde{E}_{8}$, or $\tilde{E}_{7}$. The last two possibilities can be ruled out by the fact that $N_{21}, N_{31}, N_{43}$ are 2 -sections of the fibration. The third possibility also can be ruled out; on the Jacobian surface $J(S)$ the irreducible components of singular fibres and a section would generate a sublattice of $P i c J(S)$ isomorphic to $U+E_{8}+A_{8}$, which is absurd, because $\operatorname{det}\left(U+E_{8}+A_{8}\right)(=9)$ can't be a multiple of $\operatorname{det} \operatorname{Pic} J(S)(=4$ or 16). Finally the second possibility $\tilde{A}_{7}$ also can be ruled out by considering the 4 -section $V_{2}$ and another elliptic fibration defined by the divisor

$$
V_{1}+N_{11}+H_{1}+N_{14}+V_{4}+N_{44}+H_{4}+N_{43}+V_{3}+N_{23}+H_{2}+N_{21}
$$

of type $\tilde{A}_{11}$. This proves that the other fibre is $\tilde{D}_{8}$. Now it is easy to see that the fibration does not have a section; the existence of a section would imply that $\operatorname{Pic}(S)$ contains as a sublattice $U \oplus E_{8} \oplus D_{8}$ whose determinant is not a multiple of $\operatorname{det} \operatorname{Pic}(S)$, a contradiction. So, the multisection index $l=2$ and, by Lemma 2.1 $\operatorname{det} \operatorname{Pic} J(S)=4$. The result now follows from Lemma 2.4 .

\section{REDUCIBILITY OF ELLIPTIC FIBRATIONS}

Recall that a positive-definite lattice $L$ (not necessarily integral) gives rise to a sphere packing in $L \otimes \mathbb{R}$ by spheres of radius $\sqrt{\mu(L)} / 2$, where $\sqrt{\mu(L)}$ is the minimal norm of $L$. The density $\Delta(L)$ is the ratio of the volume of one sphere to that of the fundamental domain of $L$, i.e. $\sqrt{\operatorname{det} L}$. The quantity $\delta(L)=\Delta(L) / \operatorname{vol}($ unit sphere) is called the center density: one has

$$
\delta(L)=(\sqrt{\mu(L)} / 2)^{r} / \sqrt{\operatorname{det} L}, \quad r=\operatorname{rank} L .
$$

In each dimension $r$ there is a bound for center density (cf. Table 1.2, [2]), known as Lindsey's bound $(r=3)$ and Rogers' bound $(r \geq 4)$. We denote this bound by $B_{r}$. 
Theorem 3.1. Let $f: S \rightarrow \mathbb{P}^{1}$ be an elliptic K3 surface with multisection index $l$ and Picard number $\rho$. Suppose there is no reducible fibre. Then $l^{2}$ divides $\operatorname{det}$ Pic $(S)$ and

$$
|\operatorname{det} \operatorname{Pic}(S)| / l^{2} \geq C_{\rho-2},
$$

where $C_{r}$ is an integer given as follows:

$$
\begin{gathered}
C_{1}=4, C_{2}=12, C_{3}=30, C_{4}=59, C_{5}=101, C_{6}=152, C_{7}=206, \\
C_{8}=250, C_{9}=278, C_{10}=283, C_{11}=266, C_{12}=233, C_{13}=191, \\
C_{14}=146, C_{15}=106, C_{16}=73, C_{17}=47, C_{18}=29
\end{gathered}
$$

Proof. The first assertion follows from Lemma 2.1. Let $M$ be the (narrow) MordellWeil lattice of the Jacobian surface $J(S)$. Then from Section 1

$$
\operatorname{rank} M=\rho-2, \quad \operatorname{det} M=|\operatorname{det} \operatorname{Pic} J(S)| .
$$

By Lemma 2.1, we have

$$
\begin{aligned}
B_{\rho-2} \geq \delta(M) & =(\sqrt{\mu(M)} / 2)^{\rho-2} / \sqrt{|\operatorname{det} \operatorname{PicJ}(S)|} \\
& \geq l / \sqrt{|\operatorname{det} \operatorname{Pic}(S)|} .
\end{aligned}
$$

Here we use the fact that $\mu(M) \geq 4$. Now we take the integer $C_{r}$ as the smallest integer not less than $1 /\left(B_{r}\right)^{2}$ and prove the second assertion.

Corollary 3.2. If $|\operatorname{det} \operatorname{Pic}(S)|<C_{\rho-2}$, then every elliptic fibration with or without a section on the K3 surface $S$ must have a reducible fibre.

Let $f: S \rightarrow C$ be an elliptic surface (with or without a section) and let $m_{v}$ be the number of irreducible components of the singular fibre $F_{v}$. We call the number $k=\Sigma_{v}\left(m_{v}-1\right)$ the index of reducibility of the fibration.

Theorem 3.3. Suppose that a K3 surface $S$ has an elliptic fibration with multisection index $l$ and index of reducibility $k$. Then $l^{2}$ divides $\operatorname{det} \operatorname{Pic}(S)$ and

$$
|\operatorname{det} \operatorname{Pic}(S)| / l^{2} \geq C_{\rho-2-k} /|\operatorname{det} T| \geq C_{\rho-2-k} / 2^{k},
$$

where $T$ is the sublattice of PicJ $(S)$ generated by irreducible components of singular fibres and a section. (So, $\operatorname{det} T$ is completely determined by the types of singular fibres, more precisely, $\operatorname{det} T=\Pi_{v} s_{v}$, where $s_{v}$ is the number of simple components of the singular fibre $F_{v}$.)

Proof. The second inequality follows from Lemma 1.1. Let $M$ be the narrow Mordell-Weil lattice of the Jacobian surface $J(S)$. Then from Section 1, Lemma 1.1 and Lemma 2.1

$$
\begin{gathered}
\operatorname{rank} M=\rho-2-k, \\
\operatorname{det} M=|\operatorname{det} P i c J(S)| \nu^{2} /|\operatorname{det} T| \leq|\operatorname{det} \operatorname{PicJ}(S)||\operatorname{det} T| \\
=|\operatorname{det} \operatorname{Pic}(S)||\operatorname{det} T| / l^{2} .
\end{gathered}
$$

Now the same argument as in the proof of Theorem 3.1 proves the first inequality. 
Remark. In the proof above we couldn't use the (full) Mordell-Weil lattice. The reason is that even though the determinant of this lattice gets in general smaller than $\operatorname{det} M$ (e.g. if $\rho-2-k \geq 11$, then there is no torsion section (Cox [4) and hence the determinant of the Mordell-Weil lattice is $\operatorname{det} M / \nu^{2}$ ), the lower bound for the minimal norm also gets smaller simultaneously.

Corollary 3.4. If $|\operatorname{det} \operatorname{Pic}(S)|<C_{\rho-2-k} / 2^{k}$, then the K3 surface $S$ can not have an elliptic fibration with index of reducibility $k$.

Corollary 3.5. Suppose that there is an elliptic fibration on a K3 surface $S$ with index of reducibility $k$ and that

$$
|\operatorname{det} \operatorname{Pic}(S)|<s^{2} C_{\rho-2-k} /|\operatorname{det} T|
$$

for some integer s. Then the multisection index of the fibration is less than $s$.

Examples. 1. K3 surfaces with $\rho=2$.

This case is not covered by the above results and is trivial in the sense that every elliptic fibration, if any, is irreducible. We remark here that a K3 surface $S$ with $\rho=2$ is elliptic if and only if $-\operatorname{det} \operatorname{Pic}(S)$ is a square number if and only if $\operatorname{Pic}(S)$ embeds in the unimodular even lattice $U$ of signature $(1,1)$. This follows from Lemma 2.1, or from the following arithmetical fact: an even lattice $L$ of signature $(1,1)$ has an isotropic element if and only if $-\operatorname{det} L$ is a square number if and only if $L$ embeds in $U$.

2. K3 surfaces with $\rho=3$.

A K3 surface $S$ with $\rho=3$ is elliptic if and only if $\operatorname{Pic}(S)$ embeds in $U \oplus(-2 m), m$ a positive integer. This follows from Lemma 2.1 and the decomposition $\operatorname{PicJ}(S)=$ $U \oplus(-2 m)$. Now let us consider a K3 surface $X$ with Picard lattice isomorphic to $L=U \oplus(-2 m)$. Such a surface exists, as $L$ embeds primitively in the K3 lattice $U^{3} \oplus E_{8}^{2}$ and the period map for K3 surfaces is surjective. It is easy to see that $X$ has an irreducible elliptic fibration if and only if $m \geq 2$. This shows that our bound $C_{1}=4$ is sharp.

3. A K3 surface with $\rho=4$ and $\operatorname{det} \operatorname{Pic}(S)=12$.

Consider a K3 surface $S$ with $\operatorname{Pic}(S)=U \oplus W$, where $W$ is generated by two elements $x, y, x^{2}=y^{2}=-4, x y=2$. The existence of $S$ can be shown in a similar manner as in the previous example. Since $W$ contains no roots, the surface $S$ admits an irreducible elliptic fibration whose Mordell-Weil group is $W$. This shows that the bound $C_{2}=12$ is sharp.

4. Jacobian Kummer surface $S$ with $\rho=17$.

This is a generic Kummer surface associated to the Jacobian of a curve of genus 2 . It is known [6] that

$$
T(S)=\langle-4\rangle \oplus U(2) \oplus U(2), \quad \operatorname{Pic}(S)=D_{8} \oplus D_{8} \oplus\langle 4\rangle .
$$

So, $\operatorname{det} \operatorname{Pic}(S)=64$ and the multisection index $l=1,2,4$, or 8 . The last possibility is ruled out, as there is no unimodular even lattice of signature $(1,16)$. The possibility $l=4$ also can be ruled out, as the discriminant group of $\operatorname{Pic}(S)$ does not contain an isotropic element of order 4. Applying Theorem 3.3 to each of the remaining 2 cases, we obtain the following lower bound for the index of reducibility $k$ :

$$
k \geq 2 \quad(l=1) ; \quad k \geq 4 \quad(l=2) .
$$


In particular, there is no irreducible fibration $(k=0)$ (whose existence was erroneously claimed in [5]). We do not know whether these bounds are sharp.

5. $S=K m\left(E \times E^{\prime}\right), E$ not isogenous to $E^{\prime}$.

There is no isotropic element of order 4 in the discriminant group of $\operatorname{Pic}(S)$, so the multisection index $l=1$ or 2 (see Proposition 2.5). If $l=1$, Oguiso's list [11] shows that the index of reducibility $k \geq 12$, while our method merely shows that $k \geq 4$. This happens mostly when $\operatorname{Pic}(S)$ contains a root sublattice of large rank (cf. [10]). For non-Jacobian fibrations with $l=2$, our method shows that $k \geq 7$.

\section{REFERENCES}

[1] S. Belcastro, Picard lattices of families of K3 surfaces, Ph.D. Thesis, University of Michigan (1997).

[2] J. Conway and N. Sloane, Sphere packings, lattices and groups, Springer-Verlag, 1988. MR 89a:11067

[3] F. Cossec and I. Dolgachev, Enriques Surfaces I, Birkhäuser, Boston, 1989. MR 90h:14052

[4] D. Cox, Mordell-Weil groups of elliptic curves over $\mathbb{C}(t)$ with $p_{g}=0$ or 1 , Duke Math. J. 49 (1982), 677-689. MR 84a:14029

[5] J. Keum, Two extremal elliptic fibrations on Jacobian Kummer surfaces, manuscripta math. 91 (1996), 369-377; erratum, 94 (1997), 543. MR 97h:14053; MR 98m:14038

[6] J. Keum, Automorphisms of Jacobian Kummer surfaces, Compositio Math. 107 (1997), 269288. MR 98e: 14039

[7] D. Morrison, On K3 surfaces with large Picard number, Invent. Math. 75 (1984), 105-121. MR 85j:14071

[8] S. Mukai, On the moduli space of bundles on K3 surfaces, I, Vector Bundles on Algebraic Varieties, Proc. Bombay Conference, 1984, Tata Inst. Fund. Research Studies No.11 (1987), 341-413. MR 88i:14036

[9] V. Nikulin, Integral symmetric bilinear forms and some of their applications, Math. USSR Izv. 14 (1980), 103-167.

[10] K. Nishiyama, The Jacobian fibrations on some K3 surfaces and their Mordell-Weil groups, Japan. J. Math. 22 (1996), 293-347. MR 97m:14037

[11] K. Oguiso, On Jacobian fibrations on the Kummer surfaces of the product of nonisogenous elliptic curves, J. Math. Soc. Japan 41 (1989), 651-680. MR 90j:14044

[12] T. Shioda, On the Mordell-Weil lattices, Com. Math. Univ. St. Pauli 39 (1990), 211-240. MR 91m:14056

[13] T. Shioda, Theory of Mordell-Weil lattices, Proc. ICM, Kyoto (1990), 473-489. MR 93k:14046

[14] E. Vinberg, The two most algebraic K3 surfaces, Math. Ann. 265 (1983), 1-21. MR 85k:14020

Department of Mathematics, Konkuk University, 93-1 Mojin-dong Kwanguin-Gu, Seoul 143-701, KOREA

E-mail address: jhkeum@kkucc.konkuk.ac.kr 